\title{
SISTEMAS DE SALUD MÓVIL INTEGRADOS: ROL DE LOS FACTORES SOCIOCULTURALES Y EL ENFOQUE DE SISTEMAS SOCIOTÉCNICO
}

\author{
Maria J. Brunette ${ }^{1, a, c}$, Walter H. Curioso $2, b, d$
}

\begin{abstract}
RESUMEN
En este artículo se discute el rol de los factores socioculturales para el fortalecimiento de los sistemas de salud móvil integrados. Se enfatiza la importancia de que toda iniciativa en salud móvil integre el análisis de los factores socioculturales durante el proceso de diseño e implementación de programas e intervenciones de investigación. El enfoque sociocultural y sociotécnico consideran, además, el factor humano, así como los condicionantes del entorno y el contexto, más allá de los recursos tecnológicos del sistema de salud móvil y la infraestructura que lo acoge. Metodologías como estudios observacionales, grupos focales, y entrevistas a profundidad deberían ser incluidas, idealmente, en todo proyecto en salud móvil. Asimismo, se presenta un estudio de caso en la zona norte de Lima (Perú), adaptando la plataforma de investigación participativa basada en la comunidad (CBPR, por sus siglas en inglés) que se fundamenta en el enfoque sociotécnico y evalúa los factores socioculturales con el objetivo de optimizar el proceso de diagnóstico de la tuberculosis utilizando las tecnologías móviles en salud (salud móvil).
\end{abstract}

Palabras clave: Factores culturales; Sistemas de información; Tecnología de la información; Tuberculosis; Perú (fuente: DeCS BIREME).

\section{INTEGRATED MOBILE HEALTH SYSTEMS: ROLE OF SOCIO-CULTURAL FACTORS AND THE SOCIO-TECHNICAL SYSTEMS APPROACH}

\begin{abstract}
This paper discusses the role of socio-cultural factors in strengthening integrated mobile health systems. Emphasis is placed on the importance that any initiative in mobile health should consider the analysis of socio-cultural factors during the process of the development and implementation of programs and research interventions. The socio-cultural and socio-technical approach also consider the human factor and considers the conditions of the environment and the context beyond the technological resources of the mobile health system and the infrastructure that supports it. Methodologies such as observational studies, focus groups, and in-depth interviews should be included ideally in any mobile health study. We also present a case study in the north area of Lima (Peru), adapting the Community-Based Participatory Research platform that is based on a socio-technical approach and evaluates the socio-cultural approach with the objective to optimize the diagnosis process of tuberculosis using mobile health.
\end{abstract}

Key words: Cultural factors; Information systems; Information technology; Tuberculosis; Peru (source: MeSH NLM).

\section{LOS FACTORES SOCIOCULTURALES EN EL MARCO DE SISTEMAS DE INFORMACIÓN EN SALUD}

La salud depende de muchos factores determinantes; los métodos cualitativos nos permiten estudiar e intervenir sobre tales factores, además que hacen posible explorar las interpretaciones culturales y conocer los comportamientos, creencias, actitudes y percepciones de los problemas o condiciones de salud de un grupo o población, de una manera más apropiada e integral, comparados con considerar, solamente, los métodos cuantitativos ${ }^{(1)}$.
Diversos estudios cualitativos realizados en poblaciones vulnerables, en riesgo o en grupos excluidos en el Perú y en países en vías de desarrollo, se han realizado utilizando diversas técnicas como grupos focales, observaciones y entrevistas a profundidad ${ }^{(2-4)}$.

Un buen sistema de salud necesita un sistema de información robusto, integral e interoperable ${ }^{(5)}$. En ese contexto, implementar sistemas de información en salud implica identificar de manera clara la gestión del cambio como factor estratégico transformacional y como generador de impacto en las organizaciones de salud. Los impactos

\footnotetext{
Department of Public Health, University of Massachusetts Lowell. MA, USA.

Department of Biomedical Informatics and Medical Education, School of Medicine, University of Washington. Seattle, WA, USA.

a Ingeniera Industrial, ${ }^{\mathrm{b}}$ médico cirujano, ${ }^{\mathrm{c}}$ doctora en Ingeniería Industrial y de Sistemas, ${ }^{\mathrm{d}}$ doctor en Informática Biomédica.

Recibido: 03/05/2017 Aprobado: 12/07/2017 En línea: 29/09/2017
} 
pueden ser de liderazgo, de estructura organizacional, en la organización del trabajo, culturales e, incluso, implican la gestión del talento humano ${ }^{(5)}$.

Un enfoque sistémico para comprender y mejorar los sistemas de salud debe incorporar los factores socioculturales, inclusive los factores sociopolíticos ${ }^{(6)}$.

Los sistemas de información en salud involucran, muchas veces, procesos complejos que requieren un enfoque sistémico para entender y evaluar un sistema de salud mediante las tecnologías de información y comunicación ${ }^{(7)}$.

\section{MODELOS SOCIOCULTURALES Y SOCIOTÉCNICOS APLICADOS A SISTEMAS DE INFORMACIÓN EN SALUD}

Existen diferentes marcos conceptuales que consideran escenarios socioculturales complejos y el uso de enfoques sociotécnicos. Destacan cinco modelos que son presentados a continuación por orden de antigüedad.

En primer lugar, el modelo de Henriksen y colaboradores ${ }^{\left({ }^{8}\right)}$, aplicado inicialmente en el contexto del tratamiento oncológico, aborda:

1. Las características del proveedor individual.

2. La naturaleza o complejidad del trabajo o tarea realizada.

3. El ambiente físico donde se lleva a cabo la atención de salud.

4. Las interfaces del sistema-humano involucradas.

5. Las diversas características de la organización (social, medioambiente y de gestión).

En segundo lugar, el marco de Vincent para analizar el riesgo y la seguridad propone una jerarquía de factores que pueden influir en la práctica clínica ${ }^{(9)}$. El modelo considera: fallas latentes (decisión de gestión y procesos organizacionales); condiciones del trabajo actual (factores de base: carga laboral, supervisión, comunicación, equipamiento, conocimiento/habilidad); fallas activas (actos inseguros: omisiones, fallas de acción, fallas cognitivas, violaciones), y barreras.

En tercer lugar, el modelo SEIPS (del inglés: Systems Engineering Initiative for Patient Safety) de Carayon y colaboradores ${ }^{(10)}$, propone un modelo de ingeniería de sistemas para la seguridad en el manejo del cuidado de la salud del paciente, el cual identifica tres dominios:

1. Características de los proveedores, sus herramientas y recursos, y el entorno físico-organizacional.

2. Aspectos interpersonales y técnicos de las actividades de atención de la salud.
3. Cambios en el estado de salud o comportamiento del paciente.

Como cuarto modelo, el análisis sociotécnico interactivo de Harrison y colaboradores (11) denominado ISTA (del inglés: Interactive Socio-technical Analysis) proporciona un panorama general de las relaciones complejas, emergentes, y relacionadas entre las tecnologías de información en salud, los médicos, y los flujos de trabajo dentro de cualquier sistema de salud.

El quinto modelo es propuesto por Sittig y Singh ${ }^{(12)}$ para estudiar el diseño, desarrollo, uso, implementación y evaluación de tecnologías de información en salud. El modelo conceptual sociotécnico de Sittig y Singh estudia a un sistema de información en salud como múltiples componentes de ocho dimensiones, los cuales son no lineales, emergentes y de comportamiento dinámico que interactúan unos con otros. En este modelo, todos los componentes sociales ejercen una fuerte influencia en los componentes técnicos. Las dimensiones que se incluyen son:

1. Hardware, software e infraestructura.

2. Contenido clínico que involucra datos e información.

3. Interfaz humano-computadora.

4. Gente que interactúa de alguna manera con el sistema, incluyendo programadores e, incluso, pacientes.

5. Comunicación y procesos asistenciales.

6. Características organizacionales, cultura organizacional y políticas internas.

7. Regulaciones, incluyendo normativas y reglamentos.

8. Monitoreo y evaluación de las consecuencias de la aplicación de las tecnologías de información y comunicación.

El modelo Sittig y Singh ya ha sido aplicado en América Latina. Por ejemplo, Plazzotta et al. ${ }^{(13)}$, aplicaron dicho modelo sociotécnico para analizar la experiencia de informatización en el área clínica en el Hospital Italiano de Buenos Aires en Argentina. Según reportan los autores, el modelo resultó siendo una adecuada herramienta de evaluación de la implementación de las tecnologías de información y comunicación en salud, que permitió el análisis de las ocho dimensiones, incluso de forma retrospectiva.

\section{LOS FACTORES SOCIOCULTURALES PARA FORTALECER EL DIAGNÓSTICO Y TRATAMIENTO DE LA TUBERCULOSIS USANDO LA SALUD MÓVIL: HACIA UNA SALUD CONECTADA INTEGRALMENTE}

Son muchos los factores que podrían afectar el proceso de diagnóstico de tuberculosis, los cuales se pueden agrupar como aquellos relacionados directamente con el paciente; 
los relacionados a la infraestructura física y organizacional, y a los procesos del sistema de trabajo durante el desarrollo de atención en salud.

El comportamiento del paciente durante su acceso a los servicios de salud está vinculado a diversos factores que influyen para lograr un diagnóstico eficiente, oportuno, con calidad $\mathrm{y}$, posteriormente, un tratamiento exitoso. Sin embargo, existen muchos factores críticos dentro del sistema de salud de comunidades de escasos recursos, que demoran dicho acceso. Estos factores incluyen: instalaciones inadecuadas, pobre infraestructura física, falta de personal, procesos de trabajo incoherentes, entre otros. Además, es importante resaltar el rol de los factores socioculturales dentro del funcionamiento del sistema de salud, pues, al tratarse de poblaciones de escasos recursos (inclusive viviendo bajo estándares de pobreza extrema), por lo general se encuentran socialmente excluidas y tienen alcance limitado a los servicios de la comunidad (educación, salud, vivienda, etc.).

La comprensión de cómo se podrían mejorar estos factores de sistemas complejos es un tema de continua investigación por diversos grupos en todo el mundo (14-16). Con el apoyo de los Institutos Nacionales de Salud de los Estados Unidos, uno de los autores (MJB) lidera el proyecto "Enfoque sociotécnico de sistemas para mejorar el proceso de diagnóstico de la tuberculosis mediante el uso de las tecnologías móviles de salud", una estrategia de investigación que se basa en el uso de la tecnología de la computación móvil para optimizar el proceso de diagnóstico de la tuberculosis con el objetivo de reducir los tiempos de espera de los pacientes a ser diagnosticados y comenzar el tratamiento médico. Estos elementos del sistema de salud se alinean muy bien con el núcleo de los servicios de salud conectando la demanda de las personas, la tecnología y el proceso de oferta de salud.

Dicho proyecto sigue el modelo de la ingeniería de sistemas ampliamente utilizado en los esfuerzos de mejora de procesos de atención médica y anclado en la teoría de los sistemas sociotécnicos ${ }^{(10)}$. El modelo, básicamente, analiza las interacciones de los elementos del sistema y propone soluciones que integren aspectos sociales, culturales y técnicos ${ }^{(15-17)}$. En la Figura 1 se presenta el modelo propuesto, adaptado del modelo SEIPS propuesto por Carayon.

La unidad de análisis del estudio fue el personal de salud de la Red de Salud Lima Norte VI - Túpac Amaru en Lima (Perú) que está involucrado en el proceso de diagnóstico, tratamiento, y/o prevención de la tuberculosis. Médicos, enfermeras y técnicas salud del programa de tuberculosis conforman la base del estudio etnográfico (mapping) que se presenta en la siguiente sección (ver Estudio de caso). Uno de los objetivos fundamentales de esta investigación consistía en la mejoría del sistema de trabajo de los profesionales de salud que, consecuentemente, contribuirá con la mejoría del servicio de salud que se le ofrezca al paciente. Se espera lograr mejoras estratégicas (optimización) en los procesos del sistema de trabajo de estos profesionales (que incluyen el proceso de diagnóstico y tratamiento de la tuberculosis) (cuadro central de la Figura 1). Un objetivo a largo plazo que deriva de esta optimización consiste en "traducir" el logro de este objetivo en políticas de salud y para eso se trabajará directamente con la oficina de la Dirección de Prevención y Control de la Tuberculosis (DPCTB), del Ministerio de Salud del Perú.

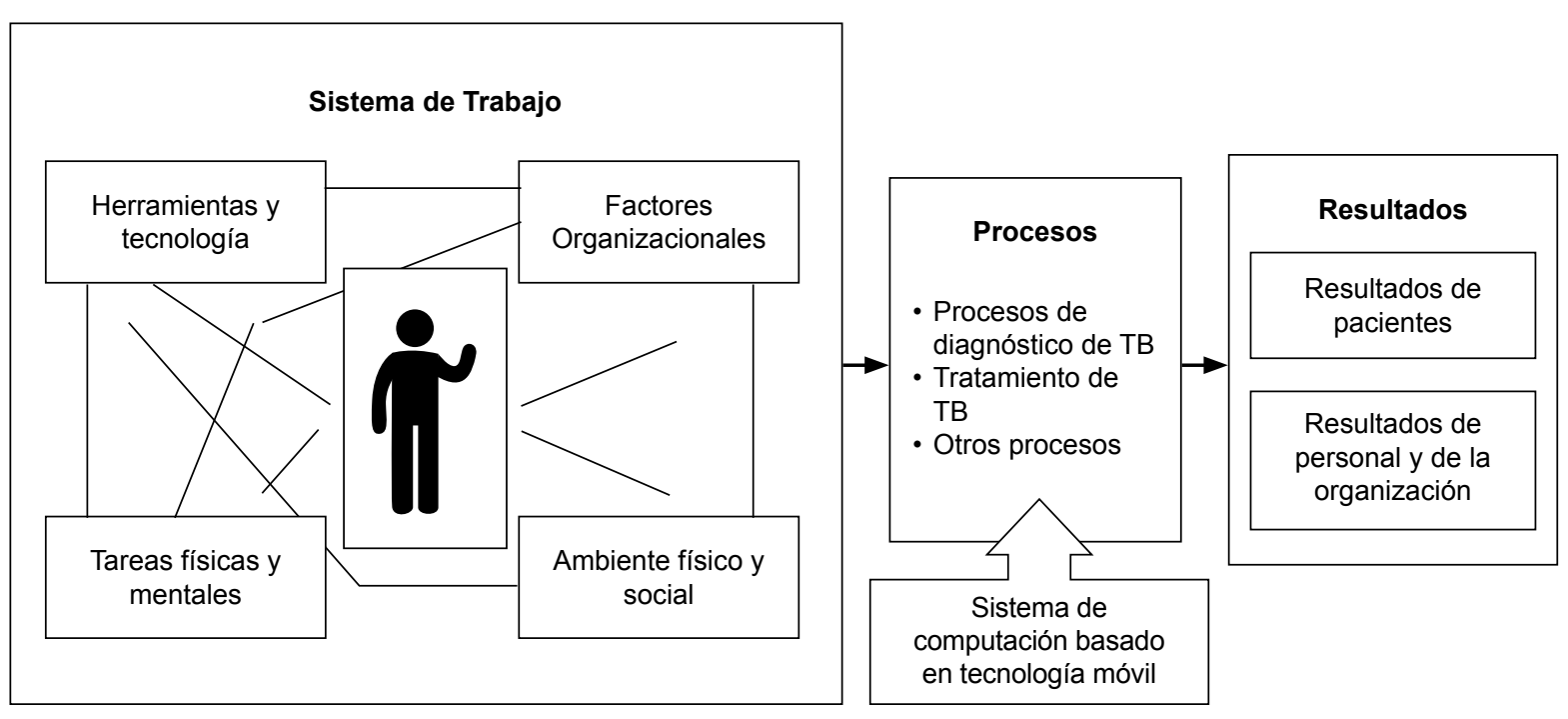

Figura 1. Modelo de un sistema sociotécnico aplicado al proceso de diagnóstico de tuberculosis. Elaboración propia. Adaptado de Carayon y colaboradores, $2006^{(10)}$. 
Nuestra propuesta sociotécnica, basada en aplicación de las tecnologías móviles aplicada a salud ${ }^{(18)}$, apunta a optimizar el proceso de diagnóstico de la tuberculosis a través de una herramienta a ser utilizada por la enfermera y/o técnica en el establecimiento de salud (EESS) directamente. Esta herramienta consiste en un dispositivo móvil que capturará imágenes de las radiografías de tórax que los pacientes se realizan en los establecimientos de salud. Dichas imágenes serán analizadas por un aplicativo dentro del dispositivo móvil y generará un resultado preliminar sobre el diagnóstico de tuberculosis. Detalles sobre el desarrollo de esta herramienta tecnológica se han presentado con anterioridad ${ }^{(19,20)}$.

\section{ESTUDIO DE CASO}

Nuestro estudio se desarrolla dentro de la plataforma de investigación participativa basada en la comunidad (o CBPR por sus siglas en inglés: Community-Based Participatory Research). Bajo el enfoque CBPR, los investigadores académicos establecen una alianza estratégica de investigación con instituciones claves dentro de la comunidad donde se realiza la investigación (gobierno local, organismos no gubernamentales, instituciones civiles, etc.). Una característica importante de este estudio, anclado en la plataforma CBPR, es que involucra de manera igualitaria a todos los miembros en el proceso de investigación y reconoce la fortaleza que cada uno aporta ${ }^{(21)}$. Entre los principios fundamentales del enfoque CBPR se pueden mencionar: 1) reconoce a la comunidad con una identidad única; 2) se basa en las fortalezas y los recursos de la propia comunidad; 3) promueve trabajo en equipo colaborativo, equitativo; 4) facilita el aprendizaje mutuo y el desarrollo de capacidades a través de un proceso de empoderamiento continuo; 5) integra y establece un balance entre la generación de conocimientos y la acción; 6) utiliza un proceso cíclico e iterativo; 7) evalúa los problemas de salud con un enfoque integral y ecológico; 8) difunde resultados a todos: la universidad, la comunidad. Se debe resaltar que los principios de CBPR necesitan ser previamente adaptados de acuerdo al contexto sociocultural de las comunidades participantes ${ }^{(22-24,26)}$.

Según se indicó, en nuestra propuesta de salud móvil resulta imprescindible el comprender cómo influyen los diversos factores socioculturales en el trabajo diario del usuario de la tecnología propuesta (médico, enfermera, técnica de enfermería). La inclusión de diversos miembros dentro del estudio, académicos y no académicos, así como un comité consultivo (CAB por sus siglas en inglés: Community Advisory Board) permiten facilitar un diálogo para considerar el rol de estos factores dentro del diseño de la solución tecnológica. La estrategia de un comité consultivo ha sido implementada exitosamente en otros programas de salud móvil a nivel internacional ${ }^{(27-29)}$.

Durante los meses de enero a abril de 2017, el análisis del sistema de salud en el proceso del diagnóstico, tratamiento y prevención de la tuberculosis fue realizado a través de la implementación de un estudio etnográfico (mapping) donde participaron enfermeras, técnicas de enfermería y médicos que laboran en diversos establecimientos de salud de la Red de Salud Túpac Amaru en Lima. La Figura 2 muestra

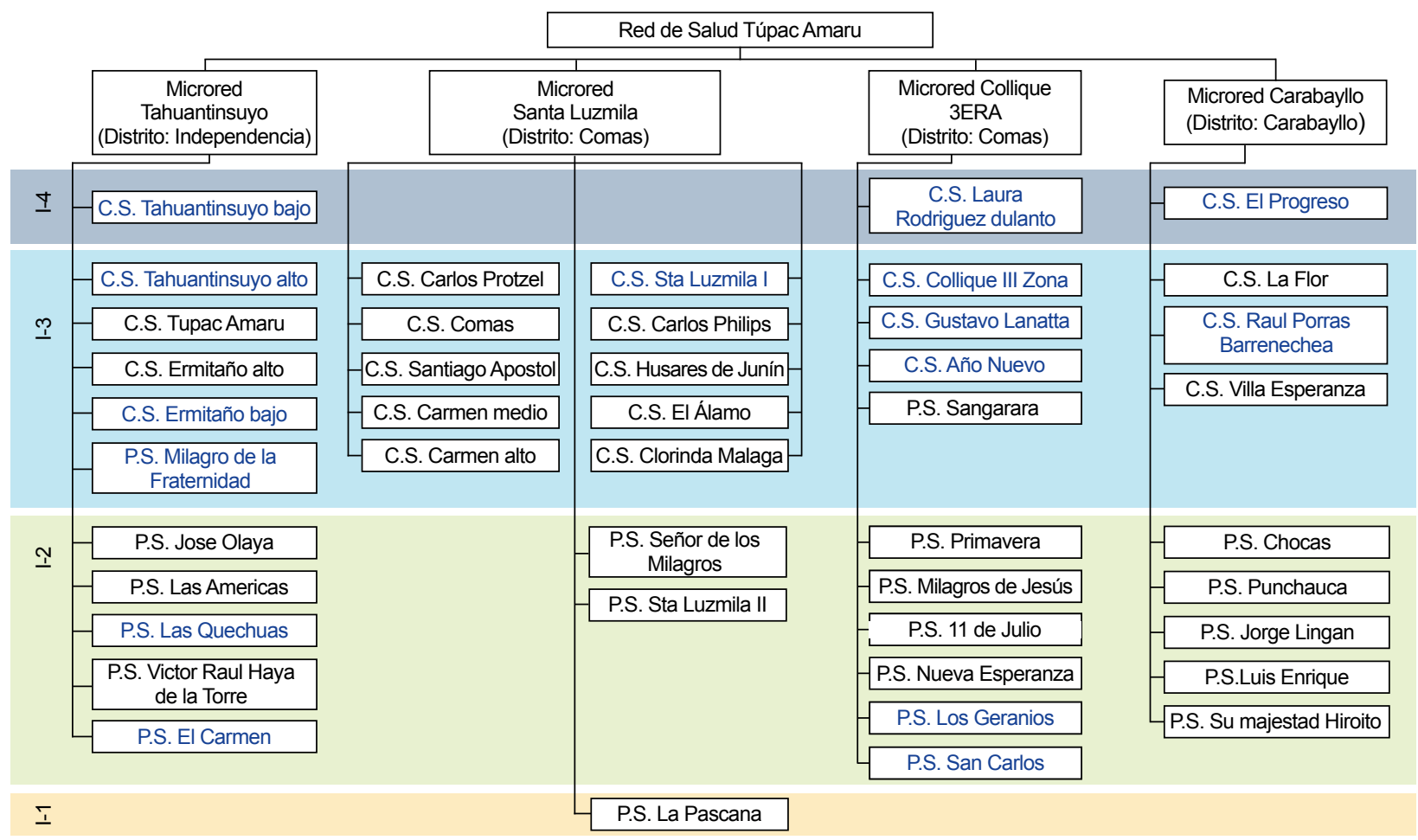

Figura 2. Establecimientos de salud participantes pertenecientes a la Red de Salud Túpac Amaru

C.S.: centro de salud; P.S.: puesto de salud 
los establecimientos de salud que participaron en el estudio (texto en color azul).

El mapeo del sistema de salud de tuberculosis estuvo estructurado en tres fases secuenciales que incluyeron estudios observacionales, entrevistas a profundidad y grupos focales con el personal de salud que pertenecen a la Red de Salud Túpac Amaru, Lima. Este mapeo obtuvo la aprobación del Comité de ética de la Universidad de Massachusetts Lowell (USA), y del Instituto Nacional de Salud (Perú). Se contó con la colaboración directa de la organización sin fines de lucro Socios en Salud Sucursal Perú para el proceso de diseño e implementación de las actividades durante el periodo indicado.

En esta investigación etnográfica, el objetivo de la investigación estaba centrado en analizar cómo funciona el sistema de trabajo del profesional de salud, es decir, cómo el médico, la enfermera y la técnica perciben su ambiente de trabajo y la organización, además de cuáles eran los retos y oportunidades que ellos encontraban en el trabajo diario. A cada uno de los participantes se les preguntó al detalle sobre las actividades de un día típico de trabajo, la carga de trabajo, la infraestructura física de su establecimiento de salud, los recursos existentes (físicos y humanos) y los diversos aspectos socioculturales de la población bajo estudio que reside en una zona urbanomarginal con elevados índices de pobreza.

Además, se trató de medir la percepción del personal de salud sobre la efectividad de los procesos y tareas específicas relacionadas a la prevención, diagnóstico y tratamiento de la tuberculosis (con un enfoque en la calidad de servicio que se le ofrece al paciente). Cabe anotar que cada participante, según se estipuló en el protocolo del estudio aprobado, participó del proceso de toma de consentimiento informado. Los protocolos y la guía de estudio que incluyen las herramientas y/o cuestionarios utilizados en el proceso del mapeo se encuentran disponibles a través de uno de los autores (MJB).

Según se indica en el esquema de la Figura 3, durante el desarrollo del mapeo se realizaron ocho estudios observacionales en cuatro establecimientos de salud de los distritos de Carabayllo, Comas e Independencia. También se realizaron 25 entrevistas a profundidad en once establecimientos de salud y, finalmente, se realizaron ocho grupos focales con participación de quince establecimientos de salud en los distritos mencionados. El análisis de los datos obtenidos en los estudios observacionales, entrevistas y grupos focales está siendo procesado actualmente, utilizando metodologías sistemáticas de análisis de datos

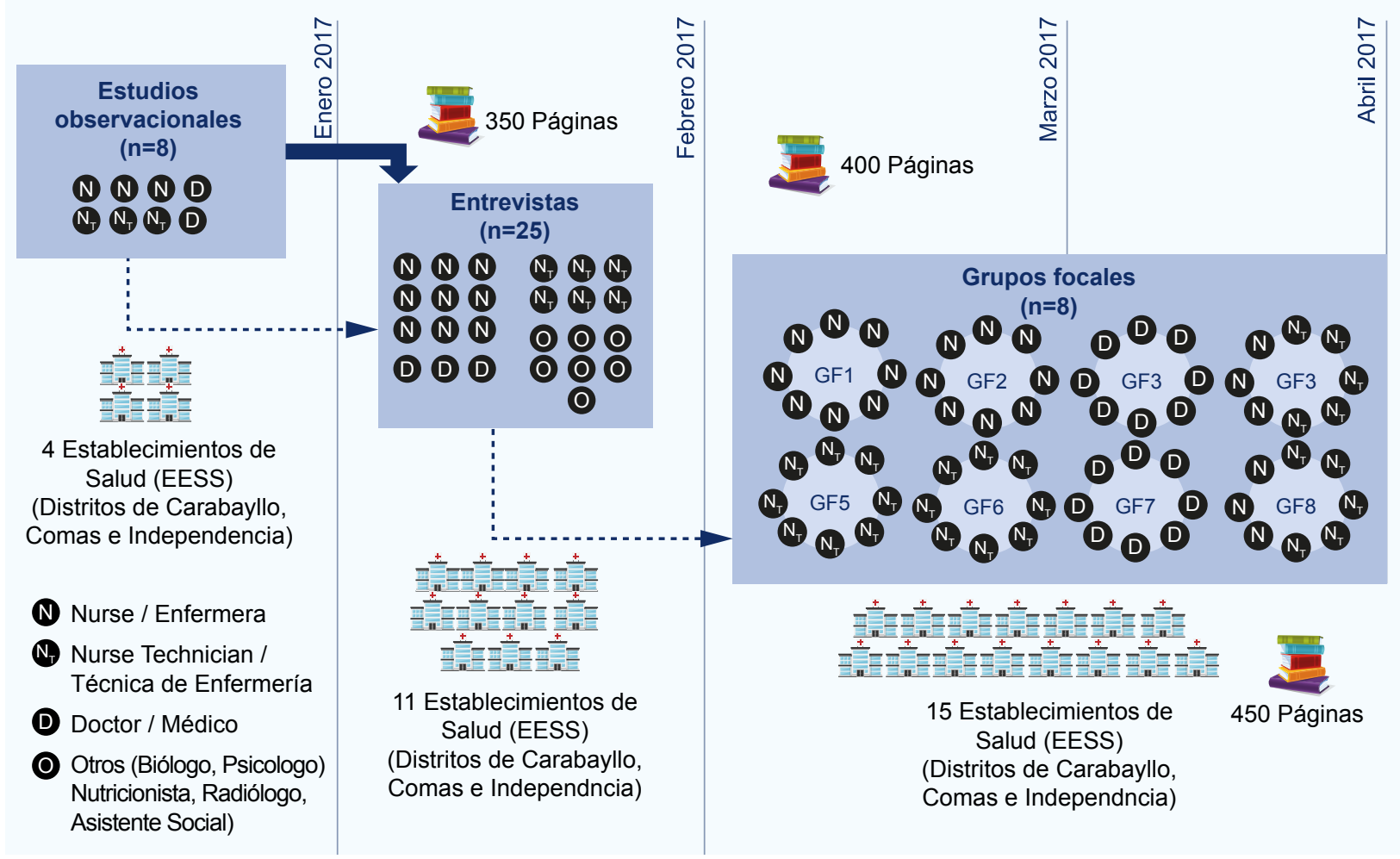

Figura 3. Mapeo del sistema de salud de Tuberculosis: proceso de recolección de data en la Red de Salud Túpac Amarú- Lima, Perú 
cualitativos (utilizando el software NVivo) y serán motivo de una publicación posterior. En forma preliminar podemos reportar que se recolectó información valiosa que nos permitirá redireccionar el diseño de la propuesta sociotecnológica (salud móvil).

Algunos de los factores que emergieron, y serán analizados luego a profundidad, incluyen: una infraestructura deficiente en algunos de los establecimientos de salud; la falta de personal de salud y la carga de trabajo elevada; la urgencia de incrementar los esfuerzos en la captación de sintomáticos respiratorios; las demoras en el proceso de diagnóstico de la tuberculosis (que incluyen demoras en la toma de las placas radiográficas y en las programaciones de las citas con el neumólogo); las demoras en el proceso de obtención del Seguro Integral de Salud y la auditoría para confirmar la cobertura del seguro; el impacto del uso de drogas y alcohol en los pacientes que abandonan el tratamiento de tuberculosis; el estigma de la enfermedad que afecta directamente a los procesos de prevención, diagnóstico y tratamiento, y la complejidad de la situación socioeconómica de la gran mayoría de los pacientes con tuberculosis.

Es importante destacar el estudio de Paz-Soldán et al. que explora e identifica los aspectos socioculturales en pacientes con tuberculosis en el Perú, como la necesidad de tener soporte social (rol de la familia y del personal de salud), el valor del soporte emocional, el estigma percibido por los pacientes con tuberculosis, el rol de las redes sociales, entre otros ${ }^{(2)}$.

En conclusión, el sistema propuesto, basado en el uso de las tecnologías móviles (salud móvil), busca optimizar las tareas de detección y diagnóstico de la tuberculosis en los diversos establecimientos de salud de la Red de Salud Túpac Amaru con el fin de reducir el tiempo de espera de los pacientes que se diagnostican e inician el tratamiento a través de la aceleración del proceso de comunicación de los resultados radiográficos. Es importante destacar que a lo largo de las fases de diseño y desarrollo de la solución técnica se han considerado, muy especialmente, los factores socioculturales y el enfoque sociotécnico a través de diversas metodologías como estudios observacionales, entrevistas a profundidad y grupos focales, para evaluar cómo dicha solución técnica (salud móvil) se va incorporando a los procesos y flujos de trabajo en los establecimientos de salud, todo con el objetivo de mejorar la eficacia y eficiencia del sistema de oferta de salud para la tuberculosis a nivel local, donde realmente se necesita focalizar y fortalecer los esfuerzos de salud pública.

Agradecimientos: este trabajo fue financiado parcialmente por el proyecto $A$ sociotechnical systems approach to improve tuberculosis diagnostics in Peru NIH/NIBIB 1R01 EB02190001 . Agradecemos la participación de los miembros del equipo de investigación, incluyendo la colaboración directa de Socios en Salud Perú, así como el apoyo de los miembros del Comité Consultivo ( $\mathrm{CAB}$ ), y en particular de los profesionales y trabajadores de salud de los establecimientos de salud dentro de la Red de Salud Túpac Amaru.

Contribuciones de autoría: MJB y WHC participaron en la redacción del manuscrito y aprobaron la versión final a publicar.

Fuentes de financiamiento: autofinanciado.

Declaración de conflictos de interés: los autores declaran no tener conflictos de interés en la publicación de este artículo.

\section{REFERENCIAS BIBLIOGRÁFICAS}

1. Ulin P, Robinson E, Tolley, E. Investigación aplicada en salud pública: métodos cualitativos. Washington, DC.: Organización Panamericana de la Salud; 2006 [citado 30 mar 2017]. Disponible en: http://apps.who.int/iris/bitstre am/10665/166144/1/9275316147.pdf

2. Paz-Soldán VA, Alban RE, Jones CD, Oberhelman RA. The provision of and need for social support among adult and pediatric patients with tuberculosis in Lima, Peru: a qualitative study. BMC Health Serv Res. 2013;13:290. doi: $10.1186 / 1472-6963-13-290$

3. Nair DM, George A, Chacko KT. Tuberculosis in Bombay: new insights from poor urban patients. Health Policy Plan. 1997;12(1):77-85.

4. Martins N, Grace J, Kelly PM. An ethnographic study of barriers to and enabling factors for tuberculosis treatment adherence in Timor Leste. Int J Tuberc Lung Dis. 2008;12(5):532-7.

5. Curioso WH. La telesalud y las nuevas fronteras de la informática biomédica en el Perú. Rev Peru Med Exp Salud Publica. 2015;32(2):217-20. doi: 10.17843/ rpmesp.2015.322.1610

6. Erazo Á. Un enfoque sistémico para comprender y mejorar los sistemas de Salud. Rev. Panam. Salud Publica. 2015; 38(3);248-53.

7. Wagenaar BH, Sherr K, Fernandes Q, Wagenaar AC. Using routine health information systems for well-designed health evaluations in low- and middle-income countries. Health Policy Plan. 2016;31(1):129-35. doi: 10.1093/ heapol/czv029
8. Henriksen K, Kaye R, Morisseau D. Industrial ergonomic factors in the radiation oncology therapy environment. En: Nielsen, R.; Jorgensen, K., editores. Advances in industrial ergonomics and safety V. Washington, DC: Taylor and Francis; 1993. p. 325-335.

9. Vincent C, Taylor-Adams S, Stanhope N. Framework for analysing risk and safety in clinical medicine. BMJ. 1998;316(7138):1154-7.

10. Carayon P, Schoofs Hundt A, Karsh BT, Gurses AP, Alvarado CJ, Smith M, et al. Work system design for patient safety: the SEIPS model. Qual Saf Health Care. 2006;15(Suppl 1):i50-8.

11. Harrison MI, Koppel R, Bar-Lev S. Unintended consequences of information technologies in health care -- an interactive sociotechnical analysis. J Am 
Med Inform Assoc. 2007;14(5):542-9. 10.1197/jamia.M2384

12. Sittig DF, Singh $H$. A new sociotechnical model for studying health information technology in complex adaptive healthcare systems. Qual Saf Health Care. 2010;19(Suppl 3):i68-74.

13. Plazzotta F, Luna D, González F. Sistemas de información en salud: integrando datos clínicos en diferentes escenarios y usuarios. Rev Peru Med Exp Salud Publica. 2015;32(2):343-51. doi: 10.17843/ rpmesp.2015.322.1630

14. Trist E. The evolution of sociotechnical systems as a conceptual framework an as an action research program. En: Van de Ven A, Joyce W, editores. Perspectives on organization design \& behavior. New York: John Wiley \& Sons; 1981.p. 19-75.

15. Carayon P, Hundt AS, Alvarado CJ, Springman S, Borgsdorf A, Jenkins L. implementing a systems engineering intervention for improving safety in outpatient surgeries. En: Henriksen K, Battles JB, Marks ES, Lewin DI, editores. Advances in patient safety: from research to implementation (volumen 3: Implementation issues). Rockville (MD): Agency for Healthcare Research and Quality (US); 2005.

16. Carayon P. Human factors of complex sociotechnical systems. Appl Ergon. 2006;37(4):525-35. doi: 10.1016/j.apergo.2006.04.011

17. Curioso WH. e-Salud en Perú: implementación de políticas para el fortalecimiento de sistemas de información en salud. Rev Panam Salud Publica. 2014;35(5-6):437-41.

18. Ruiz EF, Proaño Á, Ponce OJ, Curioso WH. Tecnologías móviles para la salud pública en el Perú: lecciones aprendidas. Rev Peru Med Exp Salud Publica. 2015;32(2):364-72. doi: 10.17843/rpmesp.2015.322.1634

19. Cao Y, Liu C, Zhang N, Liu B, Sun T, Zhang $\mathrm{P}$, et al. Improving Tuberculosis Diagnostics using deep learning and mobile health technologies among resource-poor and marginalized communities. 2016 IEEE First Conference on Connected Health: Applications, Systems and Engineering Technologies (CHASE); 27-29 de junio 2016; Washington DC, USA. Washington DC: IEEE; 2016. p. 274-81. doi: 10.1109/CHASE.2016.18

20. Alcantara MF, Cao Y, Liu C, Liu B, Brunette $\mathrm{M}$, Zhang $\mathrm{N}$, et al. Improving tuberculosis diagnostics using deep learning and mobile health technologies among resource-poor communities in Peru. Smarth Health (Amst). 2017;1-2:66-76. doi: 10.1016/j.smhl.2017.04.003

21. Brunette M, Liu B, Cao Y, Peinado J, Lecca L, Curioso W. A sociotechnical approach to improve tuberculosis diagnostics in Peru: lessons learned in first year. Póster presentado en: NSF Smart and Connected Health (SCH) PI Workshop; 21-22 marzo 2017; Boston, USA.

22. Israel BA, Schulz AJ, Parker EA, Becker $\mathrm{AB}$. Review of community-based research: assessing partnership approaches to improve public health. Annu Rev Public Health. 1998;19:173-202. doi: 10.1146/ annurev.publhealth.19.1.173

23. Wallerstein N. Empowerment to reduce health disparities. Scand J Public Health Suppl. 2002;59:72-7.

24. Wallerstein NB, Duran B. Using community-based participatory research to address health disparities. Health
Promot Pract. 2006;7(3):312-23. doi: $10.1177 / 1524839906289376$

25. Israel BA, Eng E, Schulz AJ, Parker EA, editores. Methods in community-based participatory research for health. San Francisco, CA: Jossey-Bass, 2005.

26. Minkler $M$, Wallerstein $N$, editores. Community-based participatory research for health: from process to outcomes. $2 \mathrm{da}$ ed. San Francisco, CA: Jossey-Bass, 2008.

27. Abebe NA, Capozza KL, Des Jardins TR, Kulick DA, Rein AL, Schachter AA, et al. Considerations for community-based $\mathrm{mHealth}$ initiatives: insights from three Beacon Communities. J Med Internet Res. 2013;15(10):e221. doi: 10.2196/ jmir.2803

28. Yingling LR, Brooks AT, Wallen GR, Peters-Lawrence $M$, McClurkin $M$, Cooper-McCann R, et al. Community engagement to optimize the use of webbased and wearable technology in a cardiovascular health and needs assessment study: a mixed methods approach. JMIR Mhealth Uhealth. 2016;4(2):e38. doi: 10.2196/mhealth.4489

29. Chib A, van Velthoven MH, Car J. mHealth adoption in low-resource environments: a review of the use of mobile healthcare in developing countries. J Health Commun. 2015;20(1):4-34. doi: $10.1080 / 10810730.2013 .864735$

Correspondencia: Maria J. Brunette, $P h D, M S$ Dirección: Department of Public Health, University of Massachusetts Lowell. MA, USA. Teléfono: (+978) 934-3248

Correo electrónico:maria_brunette@uml.edu 